Ferrata Storti Foundation

\title{
JUNB, DUSP2, SGK1, SOCS1 and CREBBP are frequently mutated in T-cell/histiocyte- rich large B-cell lymphoma
}

\author{
Bianca Schuhmacher, ${ }^{1}$ Julia Bein,${ }^{1}$ Tobias Rausch, ${ }^{2,3}$ Vladimir Benes ${ }^{2}$, Thomas \\ Tousseyn, ${ }^{4}$ Martine Vornanen, ${ }^{5}$ Maurilio Ponzoni, ${ }^{6}$ Lorenz Thurner, ${ }^{7,8}$ Randy \\ Gascoyne, ${ }^{9}$ Christian Steidl, ${ }^{9}$ Ralf Küppers, ${ }^{10,11}$ Martin-Leo Hansmann ${ }^{1,12,13}$ and \\ Sylvia Hartmann $n^{1,12}$
}

Haematologica 2018

Volume 104(2):330-337

\begin{abstract}
${ }^{1}$ Dr. Senckenberg Institute of Pathology, Goethe University, Frankfurt am Main, Germany; ${ }^{2}$ Genecore, European Molecular Biology Laboratory (EMBL), Heidelberg, Germany; ${ }^{3}$ Genome Biology Unit, European Molecular Biology Laboratory (EMBL), Heidelberg, Germany; ${ }^{4}$ Department of Pathology, University Hospitals K.U.Leuven, Belgium; ${ }^{5}$ Department of Pathology, Tampere University Hospital and University of Tampere, Finland; 'Unit of Lymphoid Malignancies, Department of Pathology, Scientific Institute San Raffaele, Milan, Italy; ${ }^{7}$ José Carreras Center for Immuno and Gene Therapy and Internal Medicine I, Saarland University Medical School, Homburg, Saar, Germany; ${ }^{8}$ Department of Internal Medicine 2, Hospital of the J. W. Goethe University, Frankfurt am Main, Germany; 'Department of Pathology and Laboratory Medicine and the Centre for Lymphoid Cancer, British Columbia Cancer Agency, University of British Columbia, Vancouver, Canada; ${ }^{10}$ Institute of Cell Biology (Cancer Research), Faculty of Medicine, University of DuisburgEssen, Essen, Germany; ${ }^{11}$ Deutsches Konsortium für Translationale Krebsforschung (DKTK), Germany; ${ }^{12}$ Reference and Consultant Center for Lymphoma and Lymph Node Diagnostics, Goethe University, Frankfurt am Main, Germany and ${ }^{13}$ Frankfurt Institute of Advanced Studies, Frankfurt am Main, Germany
\end{abstract}

\section{ABSTRACT}

\section{Correspondence:}

s.hartmann@em.uni-frankfurt.de

Received: August 1, 2018.

Accepted: September 7, 2018.

Pre-published: September 13, 2018.

doi:10.3324/haematol.2018.203224

Check the online version for the most updated information on this article, online supplements, and information on authorship \& disclosures: www. haematologica.org/content/104/2/330

(C)2019 Ferrata Storti Foundation

Material published in Haematologica is covered by copyright. All rights are reserved to the Ferrata Storti Foundation. Use of published material is allowed under the following terms and conditions:

https://creativecommons.org/licenses/by-nc/4.0/legalcode. Copies of published material are allowed for personal or internal use. Sharing published material for non-commercial purposes is subject to the following conditions:

https://creativecommons.org/licenses/by-nc/4.0/legalcode, sect. 3. Reproducing and sharing published material for commercial purposes is not allowed without permission in writing from the publisher.

\section{T} -cell/histiocyte-rich large B-cell lymphoma is a rare aggressive lymphoma showing histopathological overlap with nodular lymphocyte-predominant Hodgkin lymphoma. Despite differences in tumor microenvironment and clinical behavior, the tumor cells of both entities show remarkable similarities, suggesting that both lymphomas might represent a spectrum of the same disease. To address this issue, we investigated whether these entities share mutations. Ultra-deep targeted resequencing of six typical and 11 histopathological variants of nodular lymphocyte-predominant Hodgkin lymphoma, and nine cases of T-cell/histiocyte-rich large B-cell lymphoma revealed that genes recurrently mutated in nodular lymphocyte-predominant Hodgkin lymphoma are affected by mutations at similar frequencies in T-cell/histiocyte-rich large B-cell lymphoma. The most recurrently mutated genes were JUNB, DUSP2, SGK1, SOCS1 and CREBBP, which harbored mutations more frequently in T-cell/histiocyte-rich large B-cell lymphoma and the histopathological variants of nodular lymphocyte-predominant Hodgkin lymphoma than in its typical form. Mutations in JUNB, DUSP2, SGK1 and SOCS1 were highly enriched for somatic hypermutation hotspot sites, suggesting an important role of aberrant somatic hypermutation in the generation of these somatic mutations and thus in the pathogenesis of both lymphoma entities. Mutations in JUNB are generally rarely observed in malignant lymphomas and thus are relatively specific for nodular lymphocyte-predominant Hodgkin lymphoma and T-cell/histiocyte-rich large B-cell lymphoma at such high frequencies (5/17 and 5/9 cases with JUNB mutations, respectively). Taken together, the findings of the present study further support a close relationship between T-cell/histiocyte-rich large B-cell lymphoma and nodular lymphocyte-predominant Hodgkin lymphoma by showing that they share highly recurrent genetic lesions. 


\section{Introduction}

T-cell/histiocyte-rich large B-cell lymphoma (THRLBCL) is a rare subtype of diffuse large B-cell lymphoma (DLBCL) characterized by a low fraction of tumor B cells and a cellular background rich in T cells and histiocytes. It has been classified as a separate entity of mature B-cell lymphoma since the fourth edition of the World Health Organization (WHO) classification of lymphoid neoplasms. ${ }^{1,2}$ Although it has a more aggressive clinical behavior and distinct microenvironmental composition, ${ }^{3,4}$ THRLBCL shares several clinical and pathological features with nodular lymphocyte-predominant Hodgkin lymphoma (NLPHL), a rare subtype of Hodgkin lymphoma. The similarities include a predominance of middle-aged male patients ${ }^{5}$ and a minority of tumor cells derived from germinal center B cells in an abundant microenvironment. ${ }^{6,7}$ Furthermore, a high similarity of gene expression signatures $^{4,8}$ and genomic copy number changes in the microdissected tumor cells of NLPHL and THRLBCL were found. ${ }^{.}$According to Fan et al..${ }^{10}$ NLPHL can be subdivided into six different histopathological patterns, which include two typical nodular growth patterns (A and B) and four histopathological variants (C-F). The NLPHL variant E, also called THRLBCL-like variant, shows particularly marked similarities with THRLBCL and can only be distinguished from de novo THRLBCL by the presence of typical NLPHL remnants in the same lymph node or in another lymph node simultaneously sampled. In general, histopathological NLPHL variants are associated with an advanced clinical stage and an increased relapse rate..$^{10,11}$

Data on somatic gene mutations of the tumor cells of THRLBCL are still lacking. Hence, we aimed to elucidate the relationship of THRLBCL and NLPHL through a comparison of recurrently mutated genes to obtain a more comprehensive understanding of the pathogenesis of THRLBCL.

\section{Methods}

\section{Cases}

Cases were collected based on the availability of frozen tissue at the Dr. Senckenberg Institute of Pathology, Frankfurt am Main, Germany; the Department of Pathology University Hospitals, K.U. Leuven, Belgium; the Unit of Lymphoid Malignancies, San Raffaele Scientific Institute, Milan, Italy; Tampere University Hospital and University of Tampere, Tampere, Finland; and the Department of Pathology and Laboratory Medicine and the Centre for Lymphoid Cancer, British Columbia Cancer Agency, Vancouver, Canada. The local ethics committees approved the study, and written informed consent from the donors was obtained in accordance with the Declaration of Helsinki. All cases were reviewed on a multi-head microscope by expert hematopathologists (RG, SH, MLH, and TT). Only cases meeting the diagnostic criteria of the current WHO classification for NLPHL and THRLBCL ${ }^{1,2}$ were included in the study. Coexisting NLPHL was not found in any of the THRLBCL cases. For correlative analysis, NLPHL cases were classified according to Fan et al..$^{10}$ For this purpose, the major pattern present in the frozen sample was considered. In all cases, the initial biopsies before treatment were analyzed. All cases also presented a typical immunophenotype of the tumor cells (CD20+, BCL6 ${ }^{+}, \mathrm{OCT}^{+}, \mathrm{CD} 30^{-}, \mathrm{CD} 15^{-}$, $\left.\mathrm{LMP1}^{-}\right)$. Information on the patients is provided in Table 1.

\section{Ultra-deep targeted resequencing and identification of somatic variants}

Extraction of genomic DNA from frozen tissue of cases of THRLBCL and NLPHL, ultra-deep targeted resequencing after bait-based enrichment by a custom Haloplex kit (Agilent, Santa Clara, CA, USA) and processing of sequencing reads were carried out as described previously. ${ }^{12}$ All 62 genes used for the ultra-deep targeted resequencing were selected based on mutated genes from a previous study of two clonally related composite lymphomas consisting of NLPHL and DLBCL (Online Supplementary Table S1). ${ }^{12}$ Some of these selected genes were already confirmed to be mutated in primary NLPHL without transformation into DLBCL. ${ }^{12}$ The mean coverage of the 26 cases ranged between 3500 and $8500 x$ (Online Supplementary Table S2). Non-synonymous single nucleotide variants (SNVs) were filtered for allele frequencies based on the expected tumor cell content $(0.1-10 \%)$, and the presence and somatic origin of selected variants were confirmed in a semi-nested polymerase chain reaction (PCR) approach followed by Sanger sequencing of the PCR products, as indicated in Online Supplementary Table S3. Details on validation of mutations using microdissected tumor cells and analysis of the somatic hypermutation (SHM) features of SNVs are provided in the Online Supplementary Methods section.

\section{Laser microdissection and Immunohistochemistry}

Frozen sections $(5-10 \mu \mathrm{m})$ of lymph nodes from lymphoma patients were mounted on membrane-covered slides (PALM, Zeiss, Bernried, Germany), then air-dried and fixed in acetone. Sections were stained with a mouse monoclonal anti-CD20 antibody (clone L26, Dako, Glostrup, Denmark) in 1:200 dilution for $1 \mathrm{~h}$ at room temperature. Binding of the primary antibody was visualized with the aid of the Super Sensitive ${ }^{\mathrm{TM}}$ Link-Label IHC Detection System (BioGenex, Fremont, CA, USA), and counterstaining with hematoxylin was performed. For PCR analysis, 20 single tumor cells and non-tumor cells were isolated using the PALM laser capture microdissection technique (PALM MicroBeam, Zeiss, Bernried, Germany) and collected in $20 \mu \mathrm{L}$ PCR buffer without $\mathrm{MgCl}_{2}$ (Expand High Fidelity, Roche, Grenzach, Germany) supplemented with $0.1 \%$ Triton X-100.

The immunohistochemical staining for activation-induced cytidine deaminase (AICDA) was performed on an independent series of 15 typical and 11 variant NLPHL as well as 12 THRLBCL with formalin-fixed paraffin-embedded tissue as previously described. ${ }^{13}$ The anti-AICDA antibody (clone EK2 5G9, Cell Signaling, Danvers, MA, USA) was applied in a dilution of 1:100.

\section{Results}

T-cell/histiocyte-rich large B-cell lymphoma shares recurrently mutated genes with nodular lymphocyte-predominant Hodgkin lymphoma

Ultra-deep targeted resequencing of the coding exons of 62 genes (Online Supplementary Table S1), which were found to be mutated in NLPHL in a previous study, ${ }^{12}$ was performed in the following three groups: THRLBCL $(n=9)$; typical NLPHL, patterns A/B ( $\mathrm{n}=6)$; and histopathological NLPHL variants, patterns $\mathrm{C} / \mathrm{D} / \mathrm{E}(\mathrm{n}=11)$, yielding a total number of 26 cases. The patients' characteristics are given in Table 1 . The coding exons of selected genes were analyzed for non-synonymous SNVs in order to identify mutations with a potential functional effect.

Of the 62 NLPHL-related genes, 48 (77\%) were somatically mutated in at least one of the 26 cases (Online 
Table 1. Clinical information on nodular lymphocyte-predominant Hodgkin lymphoma and T-cell/histiocyte-rich large B-cell lymphoma patients.

\begin{tabular}{|c|c|c|c|c|c|c|c|}
\hline $\begin{array}{l}\text { Case } \\
\text { number }\end{array}$ & $\begin{array}{c}\text { Former } \\
\text { case number* }\end{array}$ & Diagnosis & Fan pattern" & $\begin{array}{c}\text { Estimated tumor } \\
\text { cell content }\end{array}$ & Gender & Age & Stage \\
\hline 1 & & THRLBCL & & $15 \%$ & M & 20 & IVB \\
\hline 2 & & THRLBCL & & $5 \%$ & M & 47 & IVB \\
\hline 3 & & THRLBCL & & $10 \%$ & $\mathrm{~F}$ & 50 & IVB \\
\hline 4 & & THRLBCL & & $15 \%$ & M & 40 & IVB \\
\hline 5 & & THRLBCL & & $10 \%$ & M & 50 & n.a. \\
\hline 6 & & THRLBCL & & $1 \%$ & $\mathrm{~F}$ & 89 & IVA \\
\hline 7 & & THRLBCL & & $10 \%$ & M & 27 & IVA \\
\hline 8 & & THRLBCL & & $3 \%$ & M & 31 & IVB \\
\hline 9 & & THRLBCL & & $5 \%$ & $\mathrm{~F}$ & 75 & IIIA \\
\hline 10 & & Variant NLPHL & pattern E & $1 \%$ & M & 59 & IVB \\
\hline 11 & & Variant NLPHL & pattern E & $1 \%$ & M & 46 & IVA \\
\hline 12 & & Variant NLPHL & pattern E & $1 \%$ & $\mathrm{~F}$ & 85 & IIIB \\
\hline 13 & & Variant NLPHL & pattern E & $3 \%$ & $\mathrm{M}$ & 40 & IVB \\
\hline 14 & & Variant NLPHL & pattern E & $5 \%$ & M & 23 & IIIA \\
\hline 15 & & Variant NLPHL & pattern E & $3 \%$ & M & 46 & IVA \\
\hline 16 & 6 & Typical NLPHL & pattern A & $3 \%$ & M & 10 & IIA \\
\hline 17 & 7 & Typical NLPHL & pattern A & $1 \%$ & M & 72 & n.a. \\
\hline 18 & 8 & Typical NLPHL & pattern A & $1 \%$ & M & 64 & n.a. \\
\hline 19 & 9 & Typical NLPHL & pattern A & $3 \%$ & M & 13 & IA \\
\hline 20 & 10 & Typical NLPHL & pattern A & $1 \%$ & M & 52 & n.a. \\
\hline 21 & 11 & Typical NLPHL & pattern B & $3 \%$ & $\mathrm{~F}$ & 53 & n.a. \\
\hline 22 & 5 & $\begin{array}{l}\text { Variant NLPHL, part of } \\
\text { composite lymphoma }\end{array}$ & pattern D & $3 \%$ & M & 35 & n.a. \\
\hline 23 & & Variant NLPHL & pattern C & $1 \%$ & M & 64 & n.a. \\
\hline 24 & 12 & Variant NLPHL & pattern C & $3 \%$ & M & 15 & IIA \\
\hline 25 & 13 & Variant NLPHL & pattern D & $3 \%$ & M & 31 & IA \\
\hline 26 & 17 & Variant NLPHL & pattern D & $3 \%$ & M & 75 & n.a. \\
\hline
\end{tabular}

* These cases were included in a previous study of nodular lymphocyte-predominant Hodgkin lymphoma. ${ }^{12}$ \# Histopathological patterns were determined according to Fan et al. ${ }^{10}$ THRLBCL:T-cell/histiocyte-rich large B-cell lymphoma; NLPHL: nodular lymphocyte-predominant Hodgkin lymphoma; n.a.: Information on Ann Arbor stage was not available.

Supplementary Table S3). A total of 33 genes (53\%) were recurrently affected by mutations in at least two cases (Figure 1A). Specifically in THRLBCL, 31 genes $(50 \%)$ were mutated, and 13 of these showed recurrent mutations in at least two cases, indicating a considerable mutational overlap between THRLBCL and NLPHL. The genes with the highest overall mutation frequency $(\geq 20 \%$ of all 26 cases) were JUNB, DUSP2, SGK1, SOCS1, CREBBP, FN1 and TRRAP (Figure 1A). Mutation frequencies in JUNB, SGK1, CREBBP and TRRAP were higher, albeit not significantly so, in both THRLBCL and the NLPHL variants $\mathrm{C} / \mathrm{D} / \mathrm{E}$ than in typical NLPHL A/B (Figure $1 \mathrm{~A}$ ).

The median number of mutated genes per case was comparable between typical NLPHL (median 3.5; range, 213), histopathological NLPHL variants $\mathrm{C} / \mathrm{D} / \mathrm{E}$ (median 5.0; range, 1-11) and THRLBCL (median 4.0; range, 2-20) (Figure 1B). However, the median number of SNVs per case was slightly increased in THRLBCL (median 12; range, 2-30) when compared with that in typical NLPHL (median 7; range, 2-14) and NLPHL variants C/D/E (median 8; range, 1-20) (Figure 1C). Notably, the number of SNVs per case was specifically increased in the four genes JUNB, DUSP2, SGK1 and SOCS1 (1-5 SNVs/case) when compared to CREBBP, FN1 and TRRAP (1-2 SNVs/case), particularly in regard to the shorter coding sequence of the first four genes $(<1.3 \mathrm{~kb}$ versus $>7.3 \mathrm{~kb}$ ) (Figure 1D). The higher number of SNVs per case found in THRLBCL was, therefore, related to the higher number of SNVs in these four genes.

SOCS1 was the only gene in which mutations occurred significantly more frequently in THRLBCL than in typical NLPHL A/B (4/9 cases versus $0 / 6$ cases, respectively; $P=0.010, \chi^{2}$-test) and in NLPHL in general ( $4 / 9$ cases versus $3 / 17$ cases, respectively; $P=0.035, \chi^{2}$-test).

Furthermore, the number of SNVs per case was significantly increased in SOCS1 in THRLBCL (median 5.0; range, 3-6) when compared with that in histopathological NLPHL variants $\mathrm{C} / \mathrm{D} / \mathrm{E}$ (median 1.5 ; range, $1-2 ; P=0.048$, Mann-Whitney test). JUNB was the gene with the highest number of nonsense mutations, which were present in both NLPHL (2/15 mutations) and THRLBCL (2/12 mutations).

\section{The genes with the highest mutation frequency are targets of aberrant somatic hypermutation}

Regarding the distribution of mutations in the seven most recurrently mutated genes, SNVs were generally distributed throughout the coding sequence with no differ- 
A

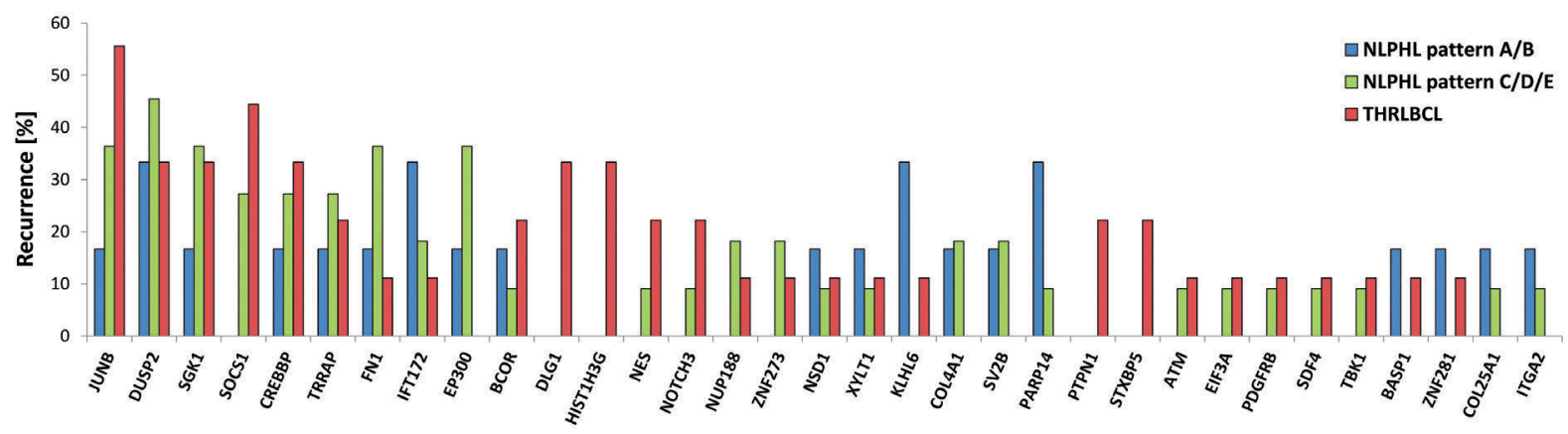

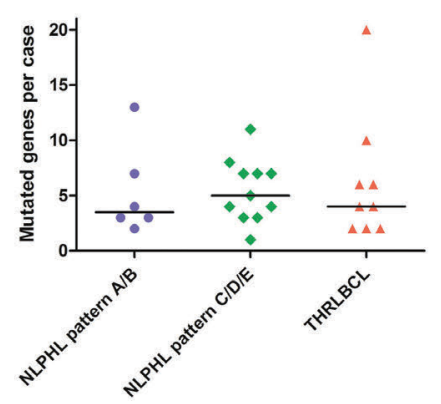

C

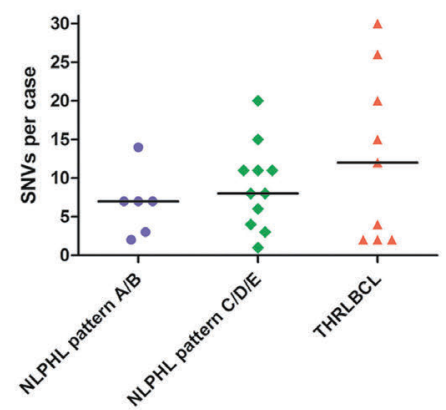

D

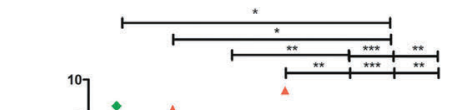

Figure 1. Genes recurrently affected by mutations and their mutational load in nodular lymphocyte-predominant Hodgkin lymphoma and T-cell/histiocyte-rich large B-cell lymphoma. (A) Frequencies of recurrently mutated genes ( $\geq 2$ cases) in six cases of NLPHL A/B (blue), 11 cases of NLPHL C/D/E (green) and nine cases of THRLBCL (red) sorted by overall recurrence. (B) Mutated genes per case in NLPHL A/B, NLPHL C/D/E and THRLBCL. (C) Number of SNVs per case in NLPHL A/B, NLPHL C/D/E and THRLBCL. (D) Scatter plot of SNVs per case and kbp of CCDS in the seven most recurrently mutated genes in NLPHL A/B, NLPHL C/D/E and THRLBCL. In (B-D), horizontal lines correspond to medians; $P$-values by Kruskal-Wallis test are indicated in the case of statistical significance. $* P<0.05$, $* \star P<0.01$, $\star * * P<0.001$. NLPHL: nodular lymphocyte-predominant Hodgkin lymphoma; THRLBCL: T-cell/histiocyte-rich large B-cell lymphoma; SNVs: single nucleotide variants; CCDS: Consensus coding sequence.

ence in the mutational distribution between the three groups of lymphoma (Figure 2). Protein domains with functional relevance were affected by mutations, e.g. the SH2 domain of the negative regulator of JAK-STAT signaling SOCS1, the catalytic domain of the protein kinase SGK1 and the histone acetyltransferase domain of the chromatin modifier CREBBP, suggesting that mutations in these genes result in an alteration of the protein function. In the three relatively small genes $(<2.5 \mathrm{~kb}) J U N B, D U S P 2$ and SOCS1, SNVs clustered to specific regions in the coding sequence with an enrichment to the first $150 \mathrm{bp}$ downstream of the start codon in JUNB, to exon 2 in DUSP2 and to the first $80 \mathrm{bp}$ of the coding sequence in SOCS1. In CREBBP, FN1 and TRRAP, which were the three larger genes ( $>75 \mathrm{~kb})$, mutations tended to be diffusely scattered and localized further downstream of the transcriptional start site. Given the fact of ongoing SHM in NLPHL and THRLBCL, ${ }^{6,7}$ as well as aberrant activity of the SHM machinery in these entities,$^{14}$ we explored whether mutations in the most recurrently mutated genes were caused by aberrant SHM. Criteria of SHM activity were investigated, as previously described in other reports. ${ }^{14-17}$ Mutations in the genes JUNB, DUSP2, SGK1 and SOCS1 were highly enriched in SHM hotspot sites (Figure $3 \mathrm{~A}$ ) and also met other SHM criteria in most SNVs (Figure 3B,C). Mutations in CREBBP, FN1 and TRRAP occasionally occurred at $C: G$ sites and showed a predominance of transitions over transversions (Figure 3B,C). However, they were clearly located outside the SHM hotspot motifs WRCY/RGYW (Figure 3A). In line with these results of an active SHM machinery and consistent with previous data, ${ }^{13,18}$ expression of AICDA could be demonstrated in the tumor cells of typical and histopathological NLPHL variants (10/15 and 3/11 cases, respectively) (Figure 3D) as well as THRLBCL (10/12 cases) (Figure 3E) in an independent case series.

\section{Discussion}

In the present study, we performed ultra-deep targeted resequencing of a set of genes that had been previously identified to be mutated in two cases of composite lymphoma of NLPHL with transformation into DLBCL. ${ }^{12}$ Four of these target genes, namely JUNB, DUSP2, SGK1 and $S O C S 1$, were also found to be mutated in primary NLPHL cases without transformation. Since the relationship of NLPHL and THRLBCL has been long-discussed, we aimed to determine whether these four genes, among others, are also mutated in THRLBCL. Here, we confirm that mutations in these genes also occur at comparable frequencies in THRLBCL. 

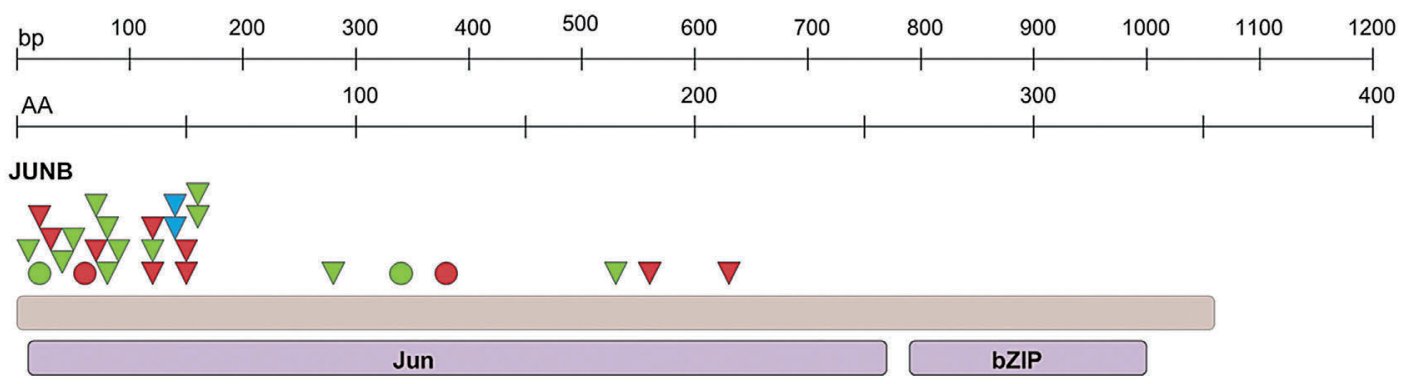

$\nabla$ missense

stop

$\square$ splice site

DUSP2

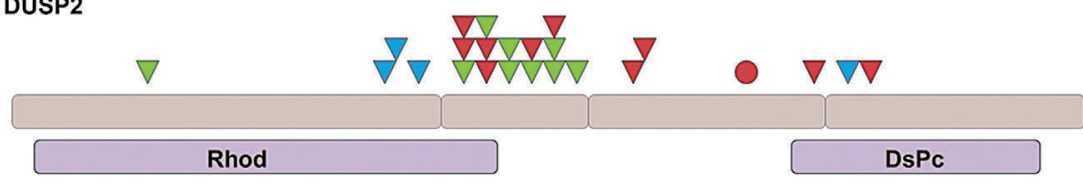

$\square$ NLPHL pattern A/B $\square$ NLPHL pattern C/D/E $\square$ THRLBCL

SGK1

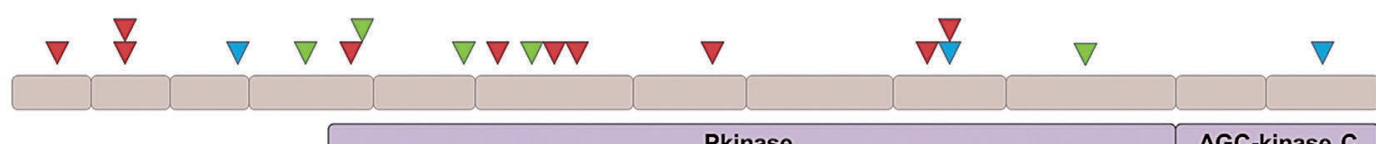

socs1
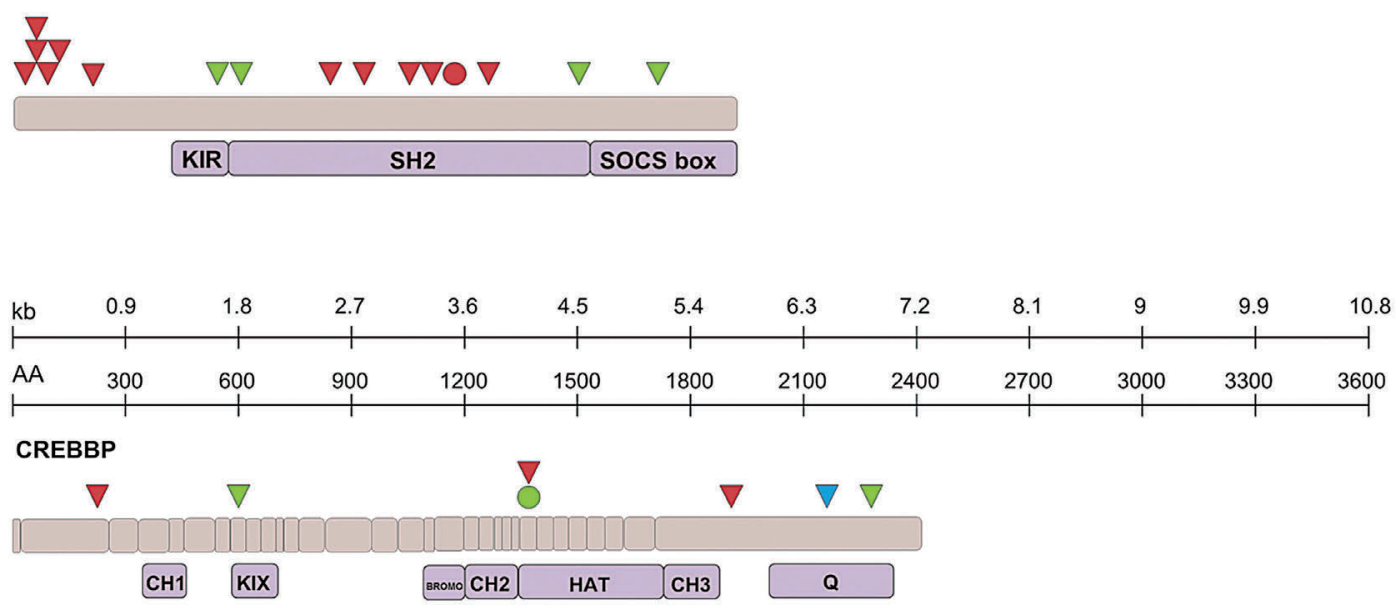

FN1

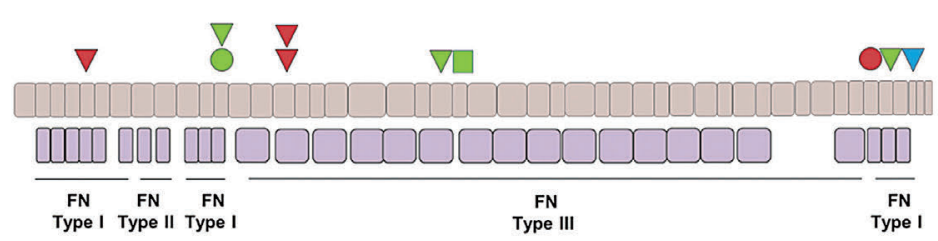

\section{TRRAP}

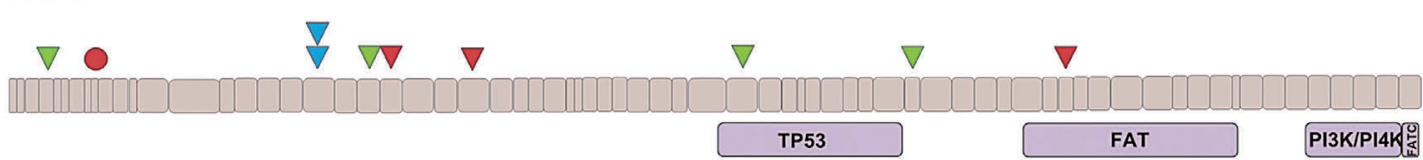

Figure 2. Mutation patterns in the most recurrently mutated genes in nodular lymphocyte-predominant Hodgkin lymphoma and T-cell/histiocyte-rich large B-cell Iymphoma. Schematic overview of the mutational distribution in the most recurrently mutated genes. For each gene missense (triangles), stop gain (spheres) and splice site (squares) mutations are mapped to coding exons (top) and protein domains (bottom). Mutations are color-coded according to the occurrence in the groups (blue: NLPHL A/B; green: NLPHL C/D/E; red: THRLBCL). Amino acid positions of protein domains are adopted from the Uniprot database (www.uniprot.org) and refer to the canonical sequences (JUNB: P17275, DUSP2: Q05923, SGK1: 000141, SOCS1: 015524, CREBBP: Q92793, FN1: P02751, TRRAP: Q9Y4A5). bZIP: basic leucine zipper motif; DsPc: dual specific phosphatase, catalytic domain; Pkinase: protein kinase domain; AGC-kinase C: AGC-kinase C-terminal domain; Rhod: rhodanese; SH2: Src homology 2 domain; KIR: kinase inhibitory region; CH1/2/3: cysteine/hystidine-rich region; KIX: kinase inducible domain; BROMO: bromodomain; HAT: histone acetyltransferase domain; Q: poly glutamine stretch; FN: fibronectin; TP53: tumor suppressor p53 binding site; FAT: FRAP-ATM-TRRAP domain; FATC: FAT C-terminal; PI3K/PI4K: phosphatidylinositol 3-kinase/ phosphatidylinositol 4-kinase domain; NLPHL: nodular lymphocyte-predominant Hodgkin lymphoma; THRLBCL: T-cell/histiocyte-rich large B-cell lymphoma. 
A

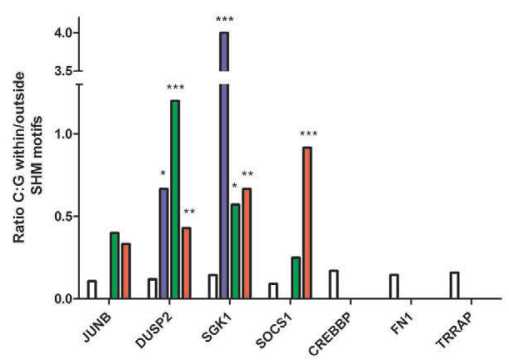

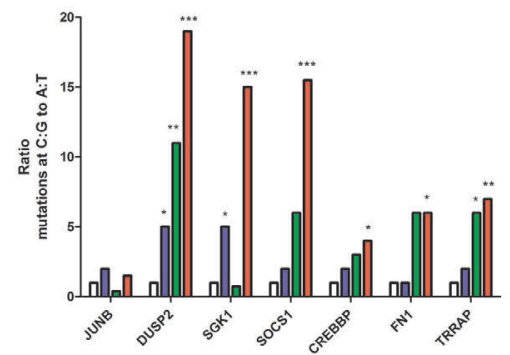

C

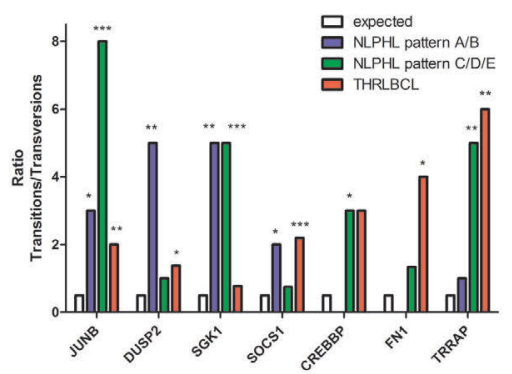

D

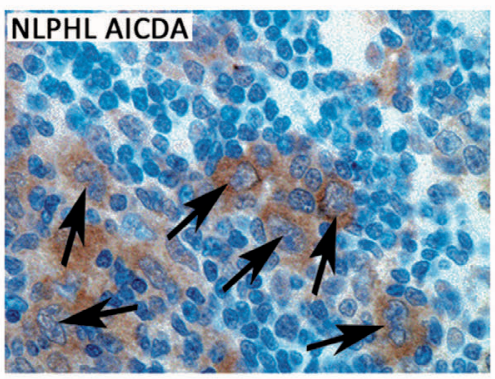

$\mathrm{E}$

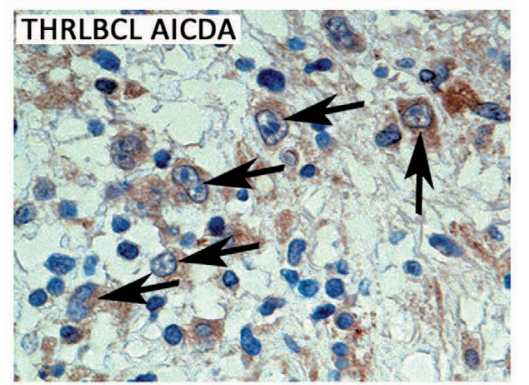

Figure 3. Characteristics of somatic mutations and activation-induced cytidine deaminase expression in nodular lymphocyte-predominant Hodgkin lymphoma and T-cell/histiocyte-rich large B-cell lymphoma. (A) Analysis of distribution of mutations to SHM hotspot motifs. (B) Ratio of mutations at C:G sites to A:T sites. (C) Ratio of transition to transversion mutations. In (A-C) synonymous SNVs were considered in addition to non-synonymous SNVs. Asterisks denote statistical significance. $* P<0.05, * * P<0.01, * * * P<0.001$. $P$-values by $\chi^{2}$ test. (D) Expression of AICDA in the LP cells of NLPHL (400x magnification). (E) Expression of AICDA in the tumor cells of THRLBCL (400x magnification). Tumor cells are highlighted by arrows. AICDA: activation-induced cytidine deaminase; NLPHL: nodular lymphocyte-predominant Hodgkin lymphoma; THRLBCL: T-cell/histiocyte-rich large B-cell lymphoma; SHM: somatic hypermutation; SNVs: single nucleotide variants.

SGK1 and SOCS1 are frequently mutated in germinal center B-cell (GCB)-derived lymphomas such as follicular lymphoma and GCB-type DLBCL. ${ }^{18-21}$ DUSP2 is less frequently mutated in malignant lymphomas; however, DUSP2 mutations have been described in DLBCL, ${ }^{15,22,23}$ primary mediastinal B-cell lymphoma ${ }^{24}$ and chronic lymphocytic leukemia. ${ }^{25}$ In contrast, to date, JUNB has been reported, to our knowledge, to be relatively specifically mutated in NLPHL. ${ }^{12}$ The total percentage of samples of hematopoietic and lymphoid neoplasms with mutations in JUNB in the COSMIC database (https://cancer.sanger.ac.uk/cosmic) is $0.09 \%$ (3 of 3516 samples tested). Two of these cases had non-synonymous mutations: one DLBCL and one lymphoid neoplasm not otherwise specified (COSMIC ID COSS2121012). The third case, a cutaneous T-cell lymphoma of mycosis fungoides type, ${ }^{26}$ presented a synonymous JUNB mutation. In the whole exome sequencing study on classical Hodgkin lymphoma by Tiacci et al. ${ }^{27}$, only one of 34 cases presented a non-synonymous JUNB mutation. Therefore, the high frequency of non-synonymous $J U N B$ mutations, including a relatively frequent occurrence of $J U N B$ nonsense mutations in both NLPHL and THRLBCL, is a hallmark of these lymphoma entities.

Mutations in the four genes JUNB, DUSP2, SGK1 and SOCS1 were more frequent in THRLBCL and the histopathological NLPHL variants than in typical NLPHL. Since we performed ultra-deep targeted resequencing of whole tissue DNA and allowed allele frequencies of mutations to range within the expected tumor cell content (0.1-
$10 \%$ ), this approach might more sensitively identify subclonal variants in THRLBCL than in NLPHL due to the slightly higher tumor cell content in THRLBCL compared to NLPHL (median 10\% versus 3\%). However, the tumor cell content did not differ significantly between histopathological NLPHL variants and typical NLPHL (3\% versus $2 \%$, respectively) and thus does not explain the higher number of mutations in histopathological NLPHL variants than in typical NLPHL.

SOCS1 is a known target of aberrant SHM, ${ }^{18}$ and SHM of immunoglobulin genes is known to be ongoing in the tumor cells of NLPHL and THRLBCL. ${ }^{6,714}$ The mutation patterns of JUNB, SGK1, DUSP2 and SOCS1 observed in the tumor cells of NLPHL and THRLBCL suggest that mutations in these four genes are most likely the result of aberrant SHM, which is likely contributing importantly to the development of these lymphomas. The genes DUSP2 and SGK1 were previously identified as potential targets of aberrant SHM in GCB-type DLBCL, ${ }^{15}$ but are less frequently mutated in GCB-type DLBCL than in NLPHL and THRLBCL. SHM activity is reported to start closely downstream from the transcriptional start site extending up to 2 $\mathrm{kb}$ into the gene, ${ }^{28}$ which is in line with an enrichment of SNVs in SHM hotspot motifs in the three relatively small genes $J U N B, D U S P 2$ and SOCS1 $(<2.5 \mathrm{~kb})$. The more frequent occurrence of SNVs in SHM hotspot sites in THRLBCL and histopathological NLPHL variants might be related to a longer and/or stronger exposure of the tumor cells to the SHM machinery. Aberrant activity of the SHM machinery may be caused by loss of target specificity and 
lead to insertion of mutations into genomic regions showing active transcription and a favorable epigenetic environment. ${ }^{15,29,30}$ This might also contribute to the more aggressive clinical behavior of these entities if a high rate of aberrant SHM affects further target genes that were not profiled here.

According to our data, SOCS1, previously shown to be mutated in NLPHL, ${ }^{12,31}$ was mutated in both histopathological NLPHL variants and THRLBCL. SOCS1 was not mutated in the typical NLPHL cases of this study, as observed previously. ${ }^{12}$ This is likely related to the low number of typical NLPHL cases investigated. On the other hand, one may speculate that SOCS1 may act as a potential driver gene indicating disease progression and may thus represent a progression driver. However, the histopathological growth patterns of the NLPHL cases investigated were not considered in the study by Mottok et al. ${ }^{31}$ Despite the fact that typical NLPHL represents the majority of NLPHL cases, in our experience most frozen NLPHL samples are acquired from histopathological NLPHL variants. One reason for this may be because the patients with histopathological NLPHL variants present with more advanced disease and are more likely to present in a specialized medical center where frozen tissue can be preserved. A potential role of SOCS1 as a driver towards disease progression is further supported by previous reports, in which SOCS1 missense mutations were found to be related to a more aggressive clinical behavior in a cohort of DLBCL cases treated with CHOP-like regimens. $^{32}$ The prognostic impact of SOCS1 mutations should therefore be investigated in a larger cohort of NLPHL. Moreover, as the ultra-deep targeted sequencing approach used here is not very reliable in the identification of structural variants, these were not considered here. Given that a high number of SOCS1 aberrations have been reported to be insertions/deletions ${ }^{31,32}$ we, therefore, likely underestimate the frequency of SOCS1 mutations.

Mutations in the acetyltransferase CREBBP were usually not a result of aberrant SHM. They frequently affected the KIX domain that mediates binding to transcription factors, ${ }^{33}$ the HAT domain that performs histone acetyltransferase activity and the C-terminal Q-rich domain, which is part of a transactivation domain, ${ }^{33}$ consistent with the functional consequences reported for DLBCL and follicular lymphoma. ${ }^{34}$ Notably, two mutations in the HAT domain of CREBBP detected in DLBCL (nonsense R1341X and $\mathrm{R} 1360 \mathrm{X})^{34}$ also occurred at the same residue in a patient with NLPHL variant pattern E (R1341X) and a patient with THRLBCL (R1360P), suggesting that the HAT domain is under inactivating pressure in these GCB-cell derived malignancies. Thus, in addition to aberrant SHM, further transforming events are likely required for the development of NLPHL and THRLBCL.

Since the gene panel applied in the present study was based on genes previously identified in composite lymphomas of NLPHL and DLBCL, we were not in a position to identify novel genes that are recurrently mutated in THRLBCL but not or only rarely in NLPHL. This is a clear limitation of our study. Thus, genome-wide mutation studies are warranted to comprehensively determine the mutational landscape of THRLBCL. Nevertheless, the fact that the most frequently mutated genes in NLPHL are also recurrently mutated in THRLBCL makes a strong point regarding the close relationship of these malignancies, which had been proposed in earlier studies based on histopathological features, related gene expression profiles, and similar genomic imbalances. ${ }^{4,8,9}$ Perhaps de novo THRLBCL could principally represent a transformation from NLPHL, and thus share key mutations that were acquired in the earlier NLPHL lymphomagenesis. Considering the clinical presentation of the patients, one could speculate that typical and histopathological NLPHL variants have a similar relationship to THRLBCL, as is observed in follicular lymphoma grade $1 / 2$ to $3 a$ and transformation into DLBCL.

\section{Acknowledgments}

The authors would like to thank Ralf Lieberz, Smaro Soworka, Nina Becker, Elena Hartung and the EMBL GeneCore sequencing team and the EMBL IT unit for their excellent technical assistance.

\section{Funding}

This project was supported by the Deutsche Forschungsgemeinschaft (grant HA6145/1-2 and HA6145/3-1).

\section{References}

1. Swerdlow SH, Campo E, Harris NL, et al. WHO Classification of Tumors of Haematopoietic and Lymphoid Tissues. 4th edition. Lyon: IARC; 2008

2. Swerdlow SH, Campo E, Harris NL, et al. WHO Classification of Tumors of Haematopoietic and Lymphoid Tissues. Revised 4th edition. Lyon: IARC; 2017.

3. Van Loo P, Tousseyn T, Vanhentenrijk V, et al. T-cell/histiocyte-rich large B-cell lymphoma shows transcriptional features suggestive of a tolerogenic host immune response. Haematologica. 2010;95(3):440-448.

4. Hartmann S, Döring C, Jakobus C, et al. Nodular lymphocyte predominant Hodgkin lymphoma and $\mathrm{T}$ cell/histiocyte rich large $B$ cell lymphoma--endpoints of a spectrum of one disease? PloS One. 2013;8(11):e78812
5. Achten R, Verhoef G, Vanuytsel L, De Wolf-Peeters C. Histiocyte-rich, T-cell-rich B-cell lymphoma: a distinct diffuse large Bcell lymphoma subtype showing characteristic morphologic and immunophenotypic features. Histopathology. 2002;40(1):31-45.

6. Braeuninger A, Küppers R, Strickler JG, Wacker HH, Rajewsky K, Hansmann ML. Hodgkin and Reed-Sternberg cells in lymphocyte predominant Hodgkin disease represent clonal populations of germinal center-derived tumor B cells. Proc Natl Acad Sci U S A. 1997;94(17):9337-9342.

7. Bräuninger A, Küppers R, Spieker T, et al. Molecular analysis of single B cells from $\mathrm{T}$ cell-rich B-cell lymphoma shows the derivation of the tumor cells from mutating germinal center $B$ cells and exemplifies means by which immunoglobulin genes are modified in germinal center B cells. Blood. 1999;93(8):2679-2687.

8. Brune V, Tiacci E, Pfeil I, et al. Origin and pathogenesis of nodular lymphocyte-predominant Hodgkin lymphoma as revealed by global gene expression analysis. J Exp Med. 2008;205(10):2251-2268.

9. Hartmann S, Döring C, Vucic E, et al. Array comparative genomic hybridization reveals similarities between nodular lymphocyte predominant Hodgkin lymphoma and $\mathrm{T}$ cell/histiocyte rich large B cell lymphoma. Br J Haematol. 2015;169(3):415-422.

10. Fan Z, Natkunam Y, Bair E, Tibshirani R, Warnke RA. Characterization of variant patterns of nodular lymphocyte predominant Hodgkin lymphoma with immunohistologic and clinical correlation. Am J Surg Pathol. 2003;27(10):1346-1356.

11. Hartmann S, Eichenauer DA, Plütschow A, et al. The prognostic impact of variant histology in nodular lymphocyte-predominant Hodgkin lymphoma: a report from the German Hodgkin Study Group (GHSG) Blood. 2013;122(26):4246-4252; quiz 4292. 
12. Hartmann S, Schuhmacher B, Rausch T, et al. Highly recurrent mutations of SGK1, DUSP2 and JUNB in nodular lymphocyte predominant Hodgkin lymphoma. Leukemia. 2016;30(4):844-853.

13. Greiner A, Tobollik S, Buettner M, et al. Differential expression of activationinduced cytidine deaminase (AID) in nodular lymphocyte-predominant and classical Hodgkin lymphoma. J Pathol. 2005;205(5): 541-547.

14. Liso A, Capello D, Marafioti T, et al. Aberrant somatic hypermutation in tumor cells of nodular-lymphocyte-predominant and classic Hodgkin lymphoma. Blood. 2006;108(3):1013-1020.

15. Khodabakhshi AH, Morin RD, Fejes AP, et al. Recurrent targets of aberrant somatic hypermutation in lymphoma. Oncotarget. 2012;3(11):1308-1319.

16. Pasqualucci L, Neumeister P, Goossens T, et al. Hypermutation of multiple proto-oncogenes in B-cell diffuse large-cell lymphomas. Nature. 2001;412(6844):341-346.

17. Mottok A, Woolcock B, Chan FC, et al. Genomic alterations in CIITA are frequent in primary mediastinal large B cell lymphoma and are associated with diminished MHC class II expression. Cell Rep. 2015;13 (7):1418-1431.

18. Mottok A, Renné C, Seifert M, et al. Inactivating SOCS1 mutations are caused by aberrant somatic hypermutation and restricted to a subset of B-cell lymphoma entities. Blood. 2009:114(20):4503-4506.

19. Morin RD, Mendez-Lago M, Mungall AJ, et al. Frequent mutation of histone-modifying genes in non-Hodgkin lymphoma. Nature. 2011;476(7360):298-303

20. Zhang J, Grubor V, Love CL, et al. Genetic heterogeneity of diffuse large B-cell lymphoma. Proc Natl Acad Sci U S A. 2013;110(4):1398-1403.

21. Pasqualucci L, Khiabanian H, Fangazio M et al. Genetics of follicular lymphoma transformation. Cell Rep. 2014;6(1):130140.

22. Morin RD, Assouline S, Alcaide M, et al. Genetic landscapes of relapsed and refractory diffuse large B-cell lymphomas. Clin Cancer Res. 2016;22(9):2290-2300.

23. Mareschal S, Dubois S, Viailly P-J, et al. Whole exome sequencing of relapsed/refractory patients expands the repertoire of somatic mutations in diffuse large B-cell lymphoma. Genes Chromosomes Cancer. 2016;55(3):251267.

24. Gunawardana J, Chan FC, Telenius A, et al. Recurrent somatic mutations of PTPN1 in primary mediastinal B cell lymphoma and Hodgkin lymphoma. Nat Genet. 2014;46(4):329-335.

25. Ljungström V, Cortese D, Young E, et al. Whole-exome sequencing in relapsing chronic lymphocytic leukemia: clinical impact of recurrent RPS15 mutations. Blood. 2016;127(8):1007-1016.

26. McGirt LY, Jia P, Baerenwald DA, et al. Whole-genome sequencing reveals oncogenic mutations in mycosis fungoides. Blood. 2015;126(4):508-519.
27. Tiacci E, Ladewig E, Schiavoni G, et al Pervasive mutations of JAK-STAT pathway genes in classical Hodgkin lymphoma. Blood. 2018:131(22):2454-2465.

28. Storb U, Peters A, Klotz E, et al. Cis-acting sequences that affect somatic hypermutation of Ig genes. Immunol Rev. 1998;162153-160.

29. Oian J, Wang O, Dose M, et al. B cell superenhancers and regulatory clusters recruit AID tumorigenic activity. Cell. 2014;159(7):1524-1537.

30. Wang $\mathrm{O}$, Oliveira $\mathrm{T}$, Jankovic $\mathrm{M}$, et al Epigenetic targeting of activation-induced cytidine deaminase. Proc Natl Acad Sci U S A. 2014:111(52):18667-18672.

31. Mottok A, Renné C, Willenbrock K Hansmann M-L, Bräuninger A. Somatic hypermutation of SOCS1 in lymphocytepredominant Hodgkin lymphoma is accompanied by high JAK2 expression and activation of STAT6. Blood. 2007:110(9): 3387-3390

32. Schif B, Lennerz JK, Kohler CW, et al. SOCS1 mutation subtypes predict divergent outcomes in diffuse large B-cell lymphoma (DLBCL) patients. Oncotarget. 2013:4(1):35-47.

33. Kwok RP, Lundblad JR, Chrivia JC, et al. Nuclear protein CBP is a coactivator for the transcription factor CREB. Nature. 1994;370(6486):223-226.

34. Pasqualucci L, Dominguez-Sola D, Chiarenza A, et al. Inactivating mutation of acetyltransferase genes in B-cell lymphoma. Nature. 2011;471(7337):189-195. 